\title{
Caractérisation De La Flore Et De La Végétation Ligneuse Des Terroirs Villageois De Keur Birame (Kaffrine) Et Saré Yorobana (Kolda) Au Sénégal
}

\author{
Mamadou Abdoul Ader Diedhiou \\ Université Assane Seck de Ziguinchor/Sénégal. UFR Sciences et \\ Technologies, Laboratoire d'Agroforesterie et d'Ecologie. Université de \\ Thiès, Institut Supérieur de Formation Agricole et Rurale, Bambey, Sénégal. \\ Département de Biologie végétale, Université Cheikh Anta Diop, Dakar, \\ Sénégal. Institut Sénégalais de Recherches Agricoles. Laboratoire National \\ de Recherche sur les Productions Végétales, Dakar, Sénégal

\section{Elhadji Faye} \\ Université de Thiès, Institut Supérieur de Formation \\ Agricole et Rurale, Bambey, Sénégal \\ Daouda Ngom \\ Département de Biologie Végétale, \\ Université Cheikh Anta Diop, Dakar, Sénégal \\ Saliou Fall \\ Institut Sénégalais de Recherches Agricoles. Laboratoire National de \\ Recherche sur les Productions Végétales, Dakar, Sénégal
}

Doi: 10.19044/esj.2018.v14n21p391 URL:http://dx.doi.org/10.19044/esj.2018.v14n21p391

\begin{abstract}
Vegetation in sub-Saharan countries is under heavy pressure from anthropogenic activities and climatic deterioration. This study was carried out at Keur Birame and Saré Yorobana villages, which are two edaphoclimatically contrasting localities. The aim of this study is to characterize their flora and woody vegetation. In doing so, 178 vegetation survey of $2500 \mathrm{~m}^{2}$ each were carried out on all two sites consisting of nine facies of vegetation. The results show a flora of the two soils of 93 species belonging to 80 genera and distributed to 33 botanical families. Among this richness, 78 species belong to Saré Yorobana and 45 species to Keur Birame. Except for regeneration rates $(37.30 \%$ and $94.04 \%)$, all structural parameters studied are higher at Saré Yorobana and the similarity index (0.48) indicates that the two vegetal communities are floristically different. The ligneous stems are generally young with small diameter that hides facies imbalances due to
\end{abstract}


human activities. It would require the various actors synergy to keep trees and biodiversity in the fields and terroirs.

Keywords: Characterization, vegetation, Keur Birame, Saré Yorobana

\section{Résumé}

La végétation des pays subsahariens est confrontée à une forte pression résultant des activités anthropiques et de la péjoration climatique. Cette étude réalisée à Keur Birame et Saré Yorobana, deux localités édaphoclimatiquement contrastées, a pour objectif la caractérisation de leur flore et de leur végétation ligneuse. Pour ce faire 178 relevés de végétation de 2500 $\mathrm{m}^{2}$ chacun ont été réalisés sur l'ensemble des deux sites constitués de neuf faciès de végétation. Les résultats montrent une flore des deux terroirs riche de 93 espèces appartenant à 80 genres et réparties à 33 familles botaniques avec 78 des espèces présentes à Saré Yorobana contre 45 espèces à Keur Birame. Excepté les taux de régénération (37,30\% et 94,04\%), tous les paramètres structuraux étudiés sont plus élevés à Saré Yorobana et l'indice de similarité $(0,48)$ indique que les deux communautés sont floristiquement différentes. Le peuplement est dans son ensemble jeune avec une dominance des individus à petits diamètres mais cela cache les déséquilibres dans les faciès causé par les activités anthropiques. Il faudrait une synergie des actions des différents acteurs pour garder les arbres dans les champs et préserver la biodiversité dans les terroirs.

Mots-clés : Caractérisation, végétation, Keur Birame, Saré Yorobana

\section{Introduction}

Les ligneux sont connus pour leurs contributions au cycle biogéochimique des systèmes de production par l'apport de la biomasse et/ou de la nécromasse, la création de microclimat favorable aux cultures et la protection des sols contre les érosions hydrique et éolienne (Breman et al., 1995 ; Young, 1995 cités par Sarr et al., 2014). Ils constituent également des sources alimentaires essentielles pour les animaux et les hommes (Sarr et al., 2013). Cependant, les réserves ligneuses subissent une forte pression anthropique et une péjoration climatique. Au Sénégal, le couvert forestier est passé entre 1990 et 2010 de 14649029 ha à 13384029 ha (FAO, 2010) et 1'empiétement des zones cultivées sur les savanes ne cesse de progresser dans les régions de Kolda, de Kaffrine et de Louga (MEDD, 2015). Ceci a pour conséquence, la perturbation de l'équilibre des formations naturelles, la régression de la jachère (Faye et $a l ., 2013$ ). Face à cette situation, la population rurale développe5 des stratégies adaptatives qui vont de la recherche de nouvelles terres de culture par le défrichement ou la récupération des jachères 
(Sarr et al., 2014). Ainsi, la structure des peuplements ligneux du Bassin arachidier est fortement modifiée par la pression anthropique. En effet, les formations forestières naturelles disparaissent au profit des parcs agroforestiers à dominance de Faidherbia albida, Ziziphus mauritiana, Adansonia digitata, Sclerocarya birrea, Anogeissus leiocarpus, Tamarindus indica, Cordyla pinnata et Balanites aegyptiaca (Mbow, 2008). A cela s'ajoutent les espèces qui rejettent après les défriches comme Guiera senegalensis, Combretum glutinosum, Combretum aculeatum, Combrehtum micrantum, Icacina senegalensis, Piliostigma reticulatum et Dichristachys glomerata (Mbow, 2008). Le terroir de Saré Yorobana, est recouvert de grandes étendues de "formations boisées avec une strate arborée et un recru arbustif bien développé" (de Wolf, 1998), de savanes et de vieilles jachères. Les dominantes sont: Combretum geitonophyllum, Combretum glutinosum, Piliostigma thonningii et Terminalia macroptera (Manley, 2000).

Cependant, des efforts sont consentis par divers acteurs pour la protection des écosystèmes forestiers et le maintien des arbres dans les agrosystèmes. Cette étude a pour objectif de caractériser la flore et la végétation ligneuses dans deux terroirs villageois des régions de Kaffrine et de Kolda ayant des caractéristiques pédoclimatiques contrastées.

\section{Matériel Et Méthodes}

\section{Présentation de la zone d'étude}

Cette étude a été menée dans les régions de Kaffrine (Keur Birame) ayant un climat de type soudano-sahélien et de Kolda (Saré Yorobana) ayant un climat de type soudano-guinéen (Figure 1). Les deux sites d'étude, respectivement dans les zones agroécologiques du Bassin arachidier et de la Casamance, ont été choisis à la suite d'une mission de prospection de zones pouvant abriter notre étude dans le centre et le sud du Sénégal. La région de Kolda présente une moyenne pluviométrique annuelle variant entre 1000 et $1500 \mathrm{~mm}$ et des sols ferrugineux, ferrugineux tropicaux lessivés et hydromorphes (Ndiaye, 1999). À Kaffrine, la moyenne annuelle pluviométrique est comprise entre 600 et $800 \mathrm{~mm}$ et les sols sont sableux, sablo-argileux ou argilo-sableux (Ndiaye et al., 2017). Les deux sites se caractérisent par l'alternance d'une saison pluvieuse et d'une saison sèche plus longue. Les principales activités pratiquées par la population est l'agriculture, l'élevage et le commerce.

Le choix des sites est motivé par les différences des conditions géoclimatiques et de la végétation mais également par les similarités des activités pratiquées par leur population. 


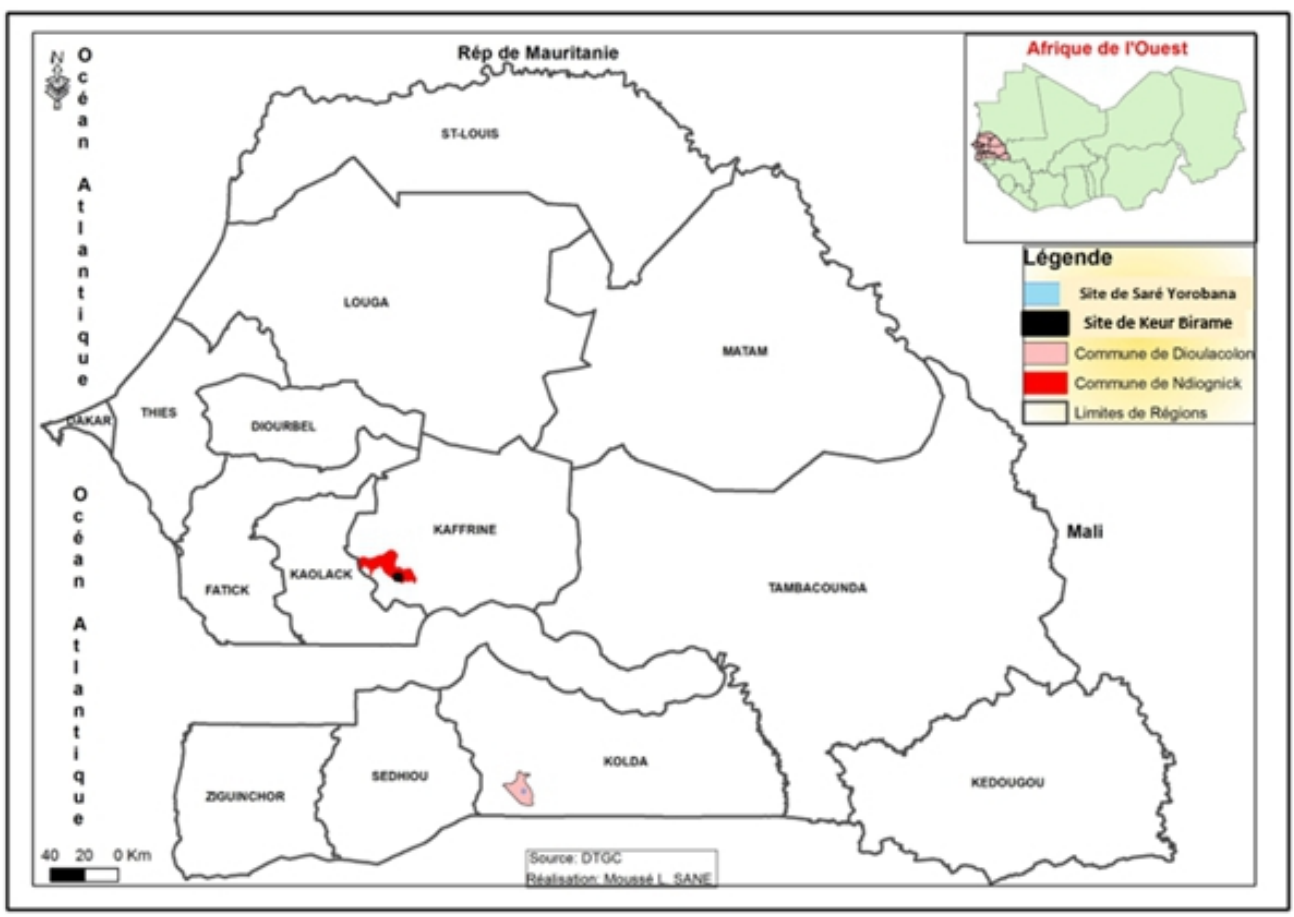

Figure 1. Carte de localisation de la zone d'étude

\section{Relevés de végétation}

L'étude a été faite sur l'étendue des terroirs villageois de Keur Birame et Saré Yorobanna constitués de neuf (9) faciès de végétation correspondant aux types d'occupation des sols rencontrés dans les deux sites renfermant des ligneux. Il a été effectué 178 relevés de végétation dans des placettes (ou placeaux) de $2500 \mathrm{~m}^{2}$ chacun dont 102 à Saré Yorobana et 76 à Keur Birame. Les relevés ont été distribués systématiquement au niveau des neuf faciès suivant des transects de direction est-ouest distants de $100 \mathrm{~m}$ avec un pas de $50 \mathrm{~m}$ entre les placettes. Cette différence du nombre d'échantillons et lié à la taille du village. Des jalons ont été utilisés pour la délimitation des placettes. Les angles droits de ces dernières sont obtenus à partir de la méthode du 3-4-5 qui repose sur le principe du théorème de Pythagore.

Toutes les espèces ligneuses rencontrées dans les placettes ont été recensées. Les mensurations sur les diamètres à hauteur de la poitrine $(1,30$ $\mathrm{m})$ et les diamètres croisés de houppier ont été faites et les régénérations ont été comptées. Les individus dont le diamètre à $1,30 \mathrm{~m}$ est inférieur à $7 \mathrm{~cm}$ (Diédhiou et al., 2014) sont considérés comme des régénérations ainsi que les palmiers dont les stipes ne font pas 1,30 m.

Le cortège floristique a été établi sur la base des relevés de végétation et la détermination des taxa effectuée à l'aide de la Flore du Sénégal (Berhaut, 1967) et de l'ouvrage Arbres, arbustes et lianes des zones séches d'Afrique de 
l'Ouest (Arbonnier, 2009). Les synonymes sont actualisés et normalisés sur la base de l'énumération des plantes à fleurs d'Afrique Tropicale (Lebrun et Stork, 1991, 1992, 1995, 1997).

\section{Traitement des données}

Les données recueillies dans les relevés de végétation ont été saisies et traitées grâce au tableur Excel et le logiciel XLStat.

L'analyse multivariée : Le logiciel XLStat a permis de faire des analyses factorielles de correspondances (AFC) qui sont l'une des principales méthodes utilisées en statistique descriptive multivariée (Baccini, 2010). L'AFC analyse les relations existantes entre deux variables qualitatives (tableau de contingence).

La richesse spécifique : Elle est évaluée à partir de la richesse spécifique totale et la richesse spécifique moyenne. La richesse spécifique totale $(\mathrm{S})$ est le nombre total d'espèces que comporte le peuplement considéré dans un écosystème donné (Ramade, 2003). La richesse spécifique moyenne correspond au nombre moyen d'espèces par relevé pour un échantillon donné (Ngom, 2013).

L'Analyse fréquentielle: L'Analyse fréquentielle est une méthode qui consiste à apprécier la distribution des espèces à travers les relevés. La fréquence de présence renseigne sur la distribution d'une espèce dans un peuplement. Elle s'exprime en pourcentage (\%), et est estimée par la formule suivante (Roberts-Pichette et Gillespie, 2002 cités par Ngom, 2013) :

$$
F=\frac{N r i}{N r} X 100
$$

$\mathrm{F}=$ fréquence de présence exprimée en pourcentage $(\%) ; \mathrm{N}_{\mathrm{ri}}=$ nombre de relevés où l'on retrouve l'espèce $\mathrm{i}$ et $\mathrm{N}_{\mathrm{r}}=$ nombre total de relevés.

La densité : La densité est le nombre d'individus par unité de surface. Elle s'exprime en nombre d'individus par hectare (Ngom, 2013). Elle est obtenue par le rapport de l'effectif total des individus dans l'échantillon par la surface échantillonnée.

$$
\operatorname{Dob}=\frac{\mathrm{N}}{\mathrm{S}}
$$

avec $D o b=$ Densité observée, $N=$ effectif total d'individus dans l'échantillon considéré et $S=$ surface de l'échantillon en ha. 
Le couvert ligneux : Le couvert ligneux est la surface de la couronne de l'arbre projetée verticalement au sol. Il est exprimé en mètre carré par hectare $\left(\mathrm{m}^{2}\right.$.ha. $\left.{ }^{-1}\right)$. Le couvert ligneux est calculé avec la formule ci-dessous :

$$
C=\frac{\sum_{i=1}^{n} \pi\left(\frac{d m h}{2}\right)^{2}}{S_{E}}
$$

avec $C=$ couvert ligneux ; $d_{m h}=$ diamètre moyen du houppier en $\mathrm{m}$; $S_{\mathrm{E}}=$ surface de l'échantillon considéré en ha.

La surface terrière : La surface terrière ou recouvrement basal désigne la surface de l'arbre évaluée à la base du tronc de l'arbre. Elle est exprimée en mètre carré par hectare $\left(\mathrm{m}^{2} \cdot\right.$ ha. $\left.^{-1}\right)$. Elle est donc obtenue à partir de la formule suivante :

$$
S_{t}=\frac{\sum_{i=1}^{n} \pi\left(\frac{d 0,3}{2}\right)^{2}}{S_{E}}
$$

Avec $S_{t}=$ surface terrière ; $d_{0,3}=$ diamètre en $\mathrm{m}$ du tronc à $0,3 \mathrm{~m} ; S_{E}=$ surface de l'échantillon considéré en ha.

Le taux de régénération du peuplement : Il est obtenu du rapport en pourcentage entre l'effectif total des jeunes plants et l'effectif total du peuplement (Poupon, 1980) :

$$
\mathrm{TRP}=\frac{\text { Effectif total des jeunes plants }}{\text { Effectif total du peuplement }} \times 100
$$

L'effectif total du peuplement regroupant aussi bien les jeunes plants que les plantes adultes.

\section{L'indice de Shannon-Weaver et l'indice de Piélou}

L'indice de diversité de Shannon-Weaver est l'indice le plus couramment utilisé dans la littérature. Il permet d'exprimer la diversité en prenant en compte le nombre d'espèces et l'abondance des individus au sein de chacune de ces espèces. L'indice de Shannon et Weaver varie de 0 à $\log _{2} S$, soit environ 4,5 pour une communauté assez riche (Frontier \& Picho-Viale, 1995). Ainsi, une communauté dominée par une seule espèce aura un coefficient moindre qu'une communauté dont toutes les espèces sont codominantes. L'indice est maximal quand tous les individus sont répartis d'une façon égale sur toutes les espèces (Frontier \& Pichod-Viale, 1995).

Il est basé sur la relation :

$$
H^{\prime}=-\sum_{i=1}^{n} P_{i} . \log _{2} P_{i} \text { où } \mathrm{p}_{\mathrm{i}} \text { est la fréquence de l'espèce } \mathrm{i} .
$$


L'indice de Shannon est souvent accompagné par l'indice d'équitabilité de Piélou (1966).

La régularité de la distribution ou équitabilité est un élément très important de la diversité car la présence d'espèces très abondante entraine mathématiquement la rareté d'autres espèces. Plus les fréquences des espèces sont équitablement réparties, plus la diversité est élevée. L'indice d'équitabilité de Pielou permet de mesurer la répartition des individus au sein des espèces, indépendamment de la richesse spécifique. Sa valeur varie de 0 (dominance d'une des espèces) à 1 (équirépartition des individus dans les espèces). La diversité maximale (Hmax) est le logarithme à base 2 du nombre total d'individus $(\mathbf{S})$.

$$
\mathbf{R}=\frac{\mathbf{H} \prime}{\mathbf{H} \max } \text { avec } \mathbf{H} \max =\log 2(\mathbf{S}) \boldsymbol{H} \max =\log _{2}(S) \text { exprimé en bit. }
$$

Indice de similarité de Dice- Sørensen : Les indices de similarité sont utilisés pour des données binaires présence/absence ou réponse positive/négative. Le coefficient de Sørensen mesure l'existence d'une communauté entre deux groupements floristiques. Il est calculé par la relation : $I_{\text {Sørensen }}=\frac{2 c}{a+b+2 c}$ où (c) représente le nombre d'espèces communes aux deux groupements $\mathbf{A}$ et $\mathbf{B}$; (a) le nombre d'espèces propres au groupement $\mathbf{A}$ et (b) le nombre d'espèces propres au groupement B. Lorsque la valeur du coefficient est supérieure à 0,5 , on considère qu'il y a une grande similarité entre les deux groupements comparés, on dit alors qu'il existe une communauté floristique entre les groupements en question (Faye, 2005).

\section{Résultats}

\section{Analyse globale de la flore ligneuse}

La flore des terroirs de Saré Yorobana et Keur Birame est riche de 93 espèces appartenant à 80 genres et réparties à 33 familles botaniques. À l'échelle du terroir, la répartition des espèces rencontrées dans les familles botaniques est présentée dans le tableau 1. Le terroir de Saré Yorobana (SY) est floristiquement plus diversifié. Il renferme 78 espèces relevant de 68 genres et de 29 familles avec en moyenne 10 espèces/relevé contre 45 espèces appartenant à 41 genres et relevant de 26 familles avec en moyenne 4 espèces/relevé pour celui de Keur Birame.

Tableau 1. Classification botanique des espèces rencontrées (+ = présence ; - = absence)

\begin{tabular}{|c|c|c|c|c|}
\hline Familles & Genres & Espèces & SY & KB \\
\hline \multirow{4}{*}{ Anacardiaceae } & Anacardium & Anacardium occidentale L. & + & - \\
\cline { 2 - 5 } & \multirow{4}{*}{ Lannea } & Lannea acida A. Rich. & + & + \\
\cline { 3 - 5 } & & Lannea velutina A. Rich. & + & - \\
\cline { 2 - 5 } & Mangifera & Mangifera indica L. & + & - \\
\hline
\end{tabular}




\begin{tabular}{|c|c|c|c|c|}
\hline & Ozoroa & Ozoroa insignis Del. & + & + \\
\hline & Sclerocarya & Sclerocarya birrea (A. Rich.) Hochst. & + & + \\
\hline & Spondias & Spondias mombin L. & + & - \\
\hline \multirow[t]{2}{*}{ Annonaceae } & Annona & Annona senegalensis Pers. & + & - \\
\hline & Hexalobus & $\begin{array}{c}\text { Hexalobus monopetalus (A. Rich.) Engl. \& } \\
\text { Diels }\end{array}$ & + & + \\
\hline \multirow[t]{4}{*}{ Apocynaceae } & Baissea & Baissea multiflora A. DC. & + & - \\
\hline & Holarrhena & Holarrhena floribunda (G. Don) Dur. \& Schinz & + & - \\
\hline & Saba & Saba senegalensis (A. DC.) Pichon & + & - \\
\hline & Strophanthus & Strophanthus sarmentosus DC. & + & + \\
\hline \multirow[t]{2}{*}{ Arecaceae } & Borassus & $\begin{array}{c}\text { Borassus akeassii Bayton, Ouédraogo \& } \\
\text { Guinko }\end{array}$ & - & + \\
\hline & Elaeis & Elaeis guineensis Jacq. & + & - \\
\hline \multirow[t]{2}{*}{ Asclepiadaceae } & Calotropis & Calotropis procera (Ait.) Ait. f. & + & + \\
\hline & Leptadenia & Leptadenia hastata (Pers.) Decne. & + & + \\
\hline Balanitaceae & Balanites & Balanites aegyptiaca (L.) Del. & - & + \\
\hline \multirow[t]{2}{*}{ Bignoniaceae } & Newbouldia & Newbouldia laevis (P. Beauv.) Seem. & + & - \\
\hline & Stereospermum & Stereospermum kunthianum Cham. & - & + \\
\hline \multirow[t]{3}{*}{ Bombacaceae } & Adansonia & Adansonia digitata $\mathrm{L}$. & + & + \\
\hline & Bombax & Bombax costatum Pellegr. \& Vuillet & + & - \\
\hline & Ceiba & Ceiba pentandra (L.) Gaertn. & - & + \\
\hline Burseraceae & Commiphora & Commiphora africana (A. Rich.) Engl. & - & + \\
\hline \multirow[t]{10}{*}{ Caesalpiniaceae } & Afzelia & Afzelia africana Smith ex Pers. & + & - \\
\hline & Bauhinia & Bauhinia reticulata Lam. & + & - \\
\hline & Cassia & Cassia sieberiana DC. & + & + \\
\hline & Cordyla & $\begin{array}{c}\text { Cordyla pinnata (Lepr. ex A. Rich.) Milne- } \\
\text { Redhead }\end{array}$ & + & + \\
\hline & Daniellia & Daniellia oliveri (Rolfe) Hutch. \& Dalz. & + & - \\
\hline & \multirow[t]{2}{*}{ Detarium } & Detarium microcarpum Guill. \& Perr. & + & - \\
\hline & & Detarium senegalense Gmel. & + & - \\
\hline & Erythrophleum & Erythrophleum africanum Afzel. & + & - \\
\hline & Piliostigma & Piliostigma reticulatum (DC.) Hochst. & + & + \\
\hline & Tamarindus & Tamarindus indica $\mathrm{L}$. & + & + \\
\hline Capparaceae & Maerua & Maerua angolensis DC. & - & + \\
\hline Celastraceae & Maytenus & Maytenus senegalensis (Lam.) Exell & + & - \\
\hline Chrysobalanaceae & Neocarya & Neocarya macrophylla (Sabine) Prance & + & - \\
\hline \multirow[t]{3}{*}{ Combretaceae } & Anogeissus & Anogeissus leiocarpus (DC.) Guill. \& Perr. & - & + \\
\hline & \multirow[t]{2}{*}{ Combretum } & Combretum geitonophylumDiels & + & - \\
\hline & & Combretum glutinosum Perr. ex DC. & + & + \\
\hline
\end{tabular}




\begin{tabular}{|c|c|c|c|c|}
\hline & & Combretum nigricans Lepr. ex Guill. et Perr. & + & - \\
\hline & Guiera & Guiera senegalensis J.F. Gmel. & + & + \\
\hline & \multirow[t]{2}{*}{ Terminalia } & Terminalia avicennioides Guill. \& Perr. & + & - \\
\hline & & Terminalia macroptera Guill. \& Perr. & + & - \\
\hline Ebenaceae & Diospyros & Diospyros mespiliformis Hochst. ex A. Rich. & - & + \\
\hline \multirow[t]{3}{*}{ Euphorbiaceae } & Euphorbia & Euphorbia balsamifera Ait. & - & + \\
\hline & Securinega & Securinega virosa (Roxb.) Baill & + & + \\
\hline & Bridelia & Bridelia micrantha (Hochst.) Baill. & + & - \\
\hline \multirow[t]{6}{*}{ Fabaceae } & Afrormosia & Afrormosia laxiflora (Benth. ex Bak.) Harms & + & - \\
\hline & Erythrina & Erythrina senegalensis DC. & + & - \\
\hline & Ormocarpum & $\begin{array}{l}\text { Ormocarpum bibracteatum (Hochst. ex A. } \\
\text { Rich.) Bak. }\end{array}$ & + & - \\
\hline & Ostryoderris & Ostryoderris stuhlmannii (Taub.) Harms & + & + \\
\hline & Pericopsis & Pericopsis laxiflora (Benth.) van Meeuwen & + & - \\
\hline & Pterocarpus & Pterocarpus erinaceus Poir. & + & - \\
\hline Hymenocardiaceae & Hymenocardia & Hymenocardia acida Tul. & + & - \\
\hline Icacinaceae & Icacina & Icacina senegalensis A. Juss. & + & - \\
\hline Loganiaceae & Strychnos & Strychnos spinosa Lam. & + & + \\
\hline \multirow[t]{2}{*}{ Meliaceae } & Azadirachta & Azadirachta indica A. Juss. & + & + \\
\hline & Khaya & Khaya senegalensis (Desr.) A. Juss. & + & - \\
\hline \multirow[t]{9}{*}{ Mimosaceae } & Albizia & Albizia chevalieri Harms & - & + \\
\hline & Dichrostachys & Dichrostachys cinerea (L.) Wight \& Arn. & + & + \\
\hline & Entada & Entada africana Guill. \& Perr. & + & - \\
\hline & Faidherbia & Faidherbia albida (Del.) Chev. & + & + \\
\hline & Parkia & Parkia biglobosa (Jacq.) R. Br. ex G. Don & + & - \\
\hline & Prosopis & Prosopis africana (Guill. \& Perr.) Taub. & + & + \\
\hline & \multirow[t]{3}{*}{ Acacia } & Acacia ataxacantha DC. & + & - \\
\hline & & Acacia macrostachya Reichenb. ex DC. & + & + \\
\hline & & Acacia seyal Del. & - & + \\
\hline \multirow[t]{4}{*}{ Moraceae } & \multirow[t]{4}{*}{ Ficus } & Ficus glumosa Delile & + & + \\
\hline & & Ficus iteophylla Miq. & + & - \\
\hline & & $\begin{array}{c}\text { Ficus sycomorus ssp. gnaphalocarpa (Miq.) } \\
\text { C.C. Berg }\end{array}$ & + & + \\
\hline & & Ficus thonningii Blume & + & + \\
\hline \multirow[t]{2}{*}{ Myrtaceae } & Eucalyptus & Eucalyptus camaldulensis Dehnh. & - & + \\
\hline & Syzygium & $\begin{array}{c}\text { Syzygium guineense var. guineense (Willd.) } \\
\text { DC. }\end{array}$ & + & - \\
\hline Poaceae & Oxytenanthera & Oxytenanthera abyssinica (A. Rich.) Munro & + & - \\
\hline Polygalaceae & Securidaca & Securidaca longipedunculata Fres. & + & + \\
\hline
\end{tabular}




\begin{tabular}{|c|c|c|c|c|}
\hline Rhamnaceae & Ziziphus & Ziziphus mauritiana Lam. & + & + \\
\hline \multirow[t]{6}{*}{ Rubiaceae } & Crossopteryx & $\begin{array}{l}\text { Crossopteryx febrifuga (Afzel. ex G. Don) } \\
\text { Benth. }\end{array}$ & + & - \\
\hline & Feretia & Feretia apodanthera Del. & - & + \\
\hline & Gardenia & Gardenia ternifolia Schumach. \& Thonn. & + & + \\
\hline & Mitragyna & Mitragyna inermis (Willd.) Kuntze & + & - \\
\hline & Pavetta & Pavetta cinereifolia Berhaut & + & - \\
\hline & Sarcocephalus & Sarcocephalus latifolius (Sm) E. A. Bruce & + & - \\
\hline Sapindaceae & Allophylus & Allophylus africanus P. Beauv. & + & - \\
\hline Simaroubaceae & Hannoa & Hannoa undulata (Guill. \& Perr.) Planch. & + & - \\
\hline \multirow[t]{3}{*}{ Sterculiaceae } & Dombeya & $\begin{array}{l}\text { Dombeya quinqueseta var. senegalensis } \\
\text { (Planch.) Keay }\end{array}$ & + & - \\
\hline & Sterculia & Sterculia setigera Del. & + & + \\
\hline & Cola & Cola cordifolia (Cav.) R. Br. & + & - \\
\hline \multirow[t]{3}{*}{ Tiliaceae } & \multirow[t]{3}{*}{ Grewia } & Grewia bicolor Juss. & - & + \\
\hline & & Grewia villosa Willd. & - & + \\
\hline & & Grewia lasiodiscus K. Schum. & + & - \\
\hline \multirow[t]{2}{*}{ Verbenaceae } & \multirow[t]{2}{*}{ Vitex } & Vitex doniana Sweet & + & - \\
\hline & & Vitex madiensis Oliv. & + & + \\
\hline
\end{tabular}

La famille dominante à Saré Yorobana (SY) est celle des Caesalpiniaceae (10 espèces) suivie de celles des Anacardiaceae (7 espèces). Les Combretaceae et les Fabaceae suivent avec 6 espèces chacune. Les autres familles sont représentées pour la majeure partie par une seule espèce. À Keur Birame (KB), la famille des Mimosaceae domine avec 6 espèces. Elle est suivie par celle des Caesalpiniaceae (4 espèces). Les Anacardiaceae, les Combretaceae et les Moraceae sont représentées par 3 espèces chacune.

\section{Distribution des espèces dans les faciès de végétation sur les deux sites}

L'analyse factorielle des correspondances effectuée sur la matrice 93 espèces x 178 relevés montre une séparation des faciès de SY de ceux de KB. L'axe F1 représente un gradient décroissant d'humidité alors l'axe F2 représente un gradient croissant d'anthropisation. En effet, on a les faciès de Keur Birame à droite qui est plus sec que SY dont les faciès se situent à gauche. Par ailleurs, les zones de plateau, de forêts, de jachère et de mise en défens sont toutes situées dans les ordonnées négatives alors que les unités cultivées et fortement anthropisées sont dans les ordonnées positives. 


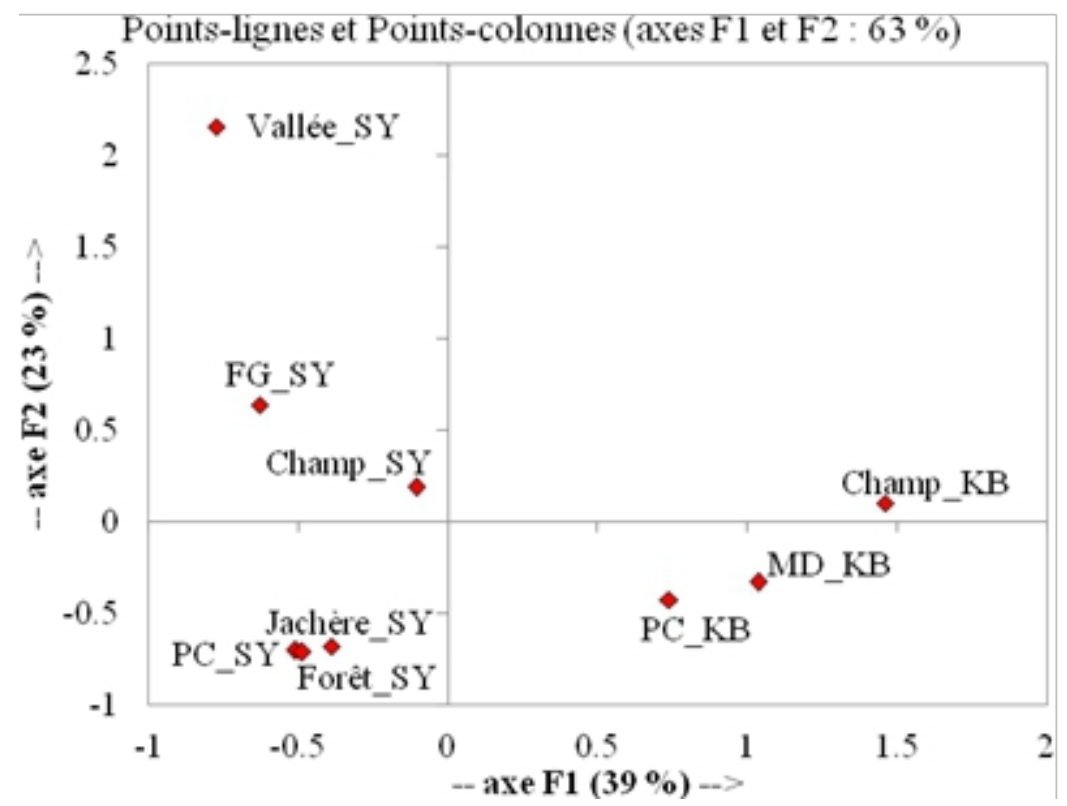

Figure 2. Analyse factorielle de correspondances de la végétation des 2 sites sur la matrice 93 espèces x 178 relevés

FG : Forêt galerie ; PC : Plateau cuirassé impropre à l'agriculture ; MD : Mise en défens ; SY : Saré Yorobana ; KB : Keur Birame.

\section{Indice de similarité}

La comparaison des deux sites par le coefficient de Sorensen $(\mathbf{C s}=$ 0,48) a montré qu'il n'existe pas de communauté floristique entre Keur Birme et Saré Yorobana. Ceci explique la distribution des faciès de végétation observée au niveau de la Figure 2.

\section{Distribution des espèces dans les faciès de végétation par terroir}

Les analyses factorielles de correspondances partielles effectuées (Figure 3) montrent la distribution des faciès dans les deux terroirs. L'AFC de Saré Yorobana, sur la matrice 78 espèces x 102 relevés (fig.3a), a révélé une répartition des faciès qui semble suivre les gradients d'humidité sur l'axe F1 et d'anthropisation sur l'axe F2. Ainsi donc, à droite, se situe la vallée avec un sol hydromorphe souvent inondée en saison des pluies. La forêt galerie (FG) qui ceinture quasiment tout le terroir villageois constitue une zone de transition entre la vallée et le reste des terres.

L'analyse factorielle de correspondances (fig.3b) sur la matrice 45 espèces x 76 relevés, a montré la distribution des espèces dans les faciès de végétation à Keur Birame. Une discrimination semble se dégager selon l'altitude (axe F1) et l'antropisation (axe F2). En effet, plus on avance sur l'axe F1 plus la zone est haute. De la même manière, sur l'axe F2, plus on 
avance plus le milieu est protégé (MD). On perd en altitude. Les axes sont ici inversement corrélés aux facteurs explicatifs.
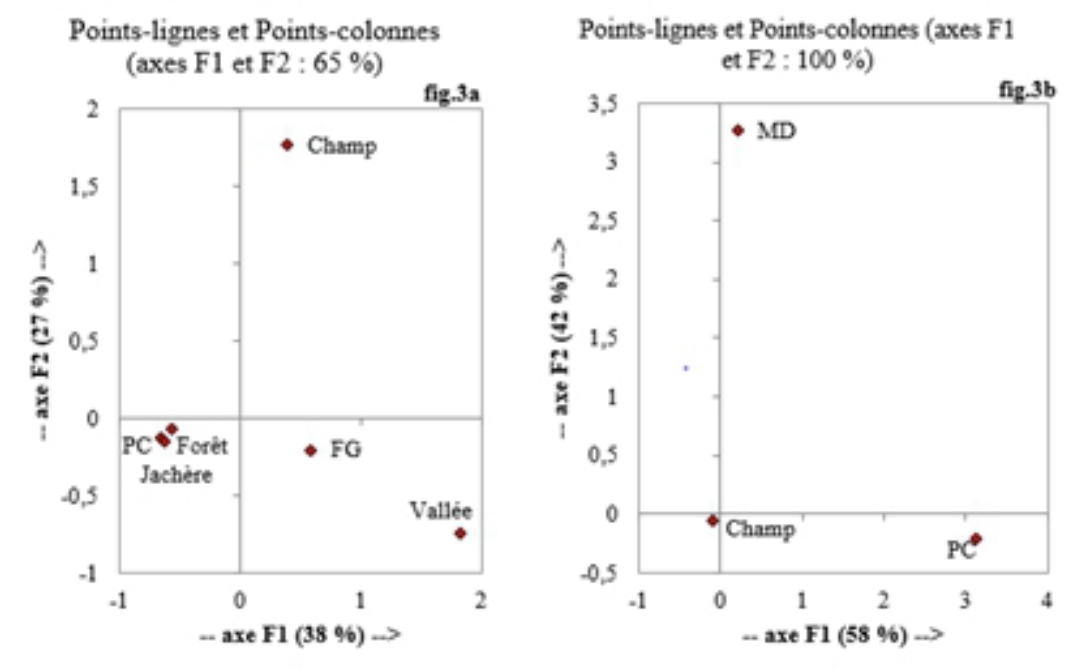

Figure 3. Analyse factorielle des correspondances de la végétation par terroir

FG : Forêt galerie ; PC : Plateau cuirassé impropre à l'agriculture ; MD : Mise en défens

\section{Indice de diversité}

D'une manière générale, comme pour la diversité spécifique, le terroir de Saré Yorobana est plus diversifié (3,99 bits) que celui de Keur Birame (2,39 bits). Le Tableau 2 montre par ailleurs qu'à Saré Yorobana la forêt galerie (FG) est le faciès le plus diversifié (4,29 bits), suivi du plateau cuirassé (PC) qui a enregistré par ailleurs le plus grand degré de réalisation de la diversité maximale $(0,42)$. La vallée est le faciès le moins diversifié mais les plus faibles niveaux de la répartition des individus dans les espèces ont été observés dans les champs et la forêt avec respectivement 0,26 et 0,27. À Keur Birame, le degré de réalisation de la diversité maximale est très faible au niveau des trois faciès. Les champs ont enregistré la plus importante diversité (2,58 bits) suivis du plateau cuirassé ( 2,10 bits).

Tableau 2. Diversité des faciès de végétation des terroirs de Saré Yorobana et Keur Birame

\begin{tabular}{|c|c|c|c|c|c|c|c|c|c|c|c|}
\hline \multirow[b]{2}{*}{ Diversité et régularité } & \multicolumn{6}{|c|}{ Faciès Saré Yoro bana } & \multicolumn{3}{|c|}{ Faciès Ke ur Birame } & \multicolumn{2}{|c|}{ Terroirs } \\
\hline & Vallée & Champ & Jachè re & FG & Forêt & PC & Chanp & MD & $\mathrm{PC}$ & SY & KB \\
\hline H' (bits) & 2,40 & 2,98 & 3,74 & 4,29 & 2,90 & 4,13 & 2,58 & 1,46 & 2,10 & 3,99 & 2,39 \\
\hline $\mathrm{H}_{\max }$ (bits) & 6,66 & 11,29 & 10,53 & 10,55 & 10,79 & 9,85 & 10,70 & 8,81 & 9,03 & 13,02 & 11,36 \\
\hline $\mathbf{R}$ & 0,36 & 0,26 & 0,36 & 0,41 & 0,27 & 0,42 & 0,24 & 0,17 & 0,23 & 0,31 & 0,21 \\
\hline
\end{tabular}

\section{Indice de similarité de Dice-Sørensen}

La comparaison des faciès de Saré Yorobanna (Tableau 3) montre qu'il n'y a pas de communauté floristique entre la vallée et les autres faciès. Par ailleurs, tous les autres faciès de végétation ont un indice de similarité supérieur à 0,5 donc appartiennent à une même communauté floristique. La 
comparaison des faciès de Keur Birame montre qu'il existe une proximité floristique entre les champs et le plateau cuirassé $(0,54)$. Tandis que la mise en défens (MD) est floristiquement différente des autres faciès de végétation.

Tableau 3. Indice de similarité de Dice-Sorensen entre faciès dans les deux terroirs

\begin{tabular}{|c|c|c|c|c|c|c|c|c|}
\hline \multicolumn{6}{|c|}{ Saré Yorobana } & \multicolumn{3}{|c|}{ Keur Birame } \\
\hline PC & 0,6 & & & & & MD & 0,47 & \\
\hline Forêt & 0,52 & 0,66 & & & & PC & 0,54 & 0,36 \\
\hline FG & 0,64 & 0,70 & 0,54 & & & & Champ & $\mathrm{MD}$ \\
\hline Jachè re & 0,66 & 0,82 & 0,67 & 0,76 & & & & \\
\hline Vallée & 0,4 & 0,27 & 0,3 & 0,48 & 0,33 & & & \\
\hline & Champ & $\mathrm{PC}$ & Forêt & FG & Jachère & & & \\
\hline
\end{tabular}

\section{Paramètres structuraux}

Le Tableau 4 fait une synthèse de quelques paramètres structuraux de la végétation des terroirs de Saré Yorobana et de Keur Birame.

Tableau 4. Quelques paramètres structuraux de la végétation de Saré Yorobana et de Keur Birame

\begin{tabular}{|c|c|c|c|c|c|c|c|c|c|c|c|}
\hline & \multicolumn{6}{|c|}{ Faciès Saré Yorobana } & \multicolumn{3}{|c|}{ Facî̀s Ke ur Birame } & \multicolumn{2}{|c|}{ Terroirs } \\
\hline Paramètres struxturaux & Vallée & Champ & Jachère & FG & Forêt & PC & Champ & MD & PC & SY & KB \\
\hline Rirhesse spécifique (espèces) & 18 & 38 & 44 & 59 & 31 & 45 & 38 & 14 & 23 & 78 & 45 \\
\hline Densité réelle (ind $\cdot \mathbf{h a}^{-1}$ ) & 67 & 264 & 348 & 286 & 590 & 410 & 102 & 450 & 299 & 322 & 139 \\
\hline Surface terrière $\left(\mathrm{nr}^{2} . \mathrm{ha}^{-1}\right)$ & 4,65 & 5,00 & 2,45 & 6,54 & 4,08 & 4,71 & 3,09 & 0,37 & 0,40 & 4,39 & 2,70 \\
\hline Taux $R e$ & 14,88 & 9,36 & 24,64 & 35,82 & 31,11 & 35,88 & 8,15 & 2,68 & 2,26 & 20,90 & 7,32 \\
\hline Taux Régénération (\%) & 23,76 & 95,78 & 96,75 & 19,60 & 1,41 & 4,23 & 91,27 & 98,67 & 98,85 & 37,30 & 94,04 \\
\hline
\end{tabular}

Tous les paramètres étudiés sont plus élevés à Saré Yorobana comparés à Keur Birame, sauf le taux de régénération.

\section{Structures de la végétation à Saré Yorobana}

La Figure 4 présente les structures de la végétation dans le terroir de Saré Yorobana en fonction des diamètres. Globalement, la végétation dégage une allure avec une structure en « $\mathrm{L}$ » synonyme d'un bon état de renouvellement des ressources végétales (fig.4a). On remarque nettement la dominance des individus de faibles diamètres de la classe $] 0-7 \mathrm{~cm}$ ] suivie de la classe $77-14 \mathrm{~cm}]$. L'importance de présence des individus diminue selon que le diamètre augmente. Exceptée la vallée (fig.4b) qui a une structure plus ou moins irrégulière, les autres faciès de végétation ont une structure en « $\mathrm{L}$ » malgré les variations de densité. 


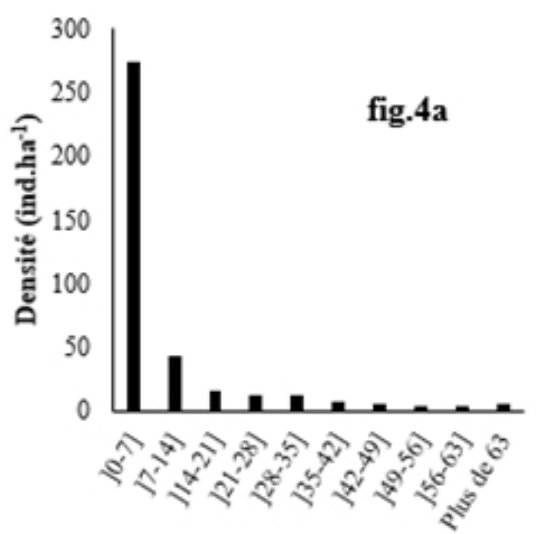

Classes de diamètres $(\mathrm{cm})$

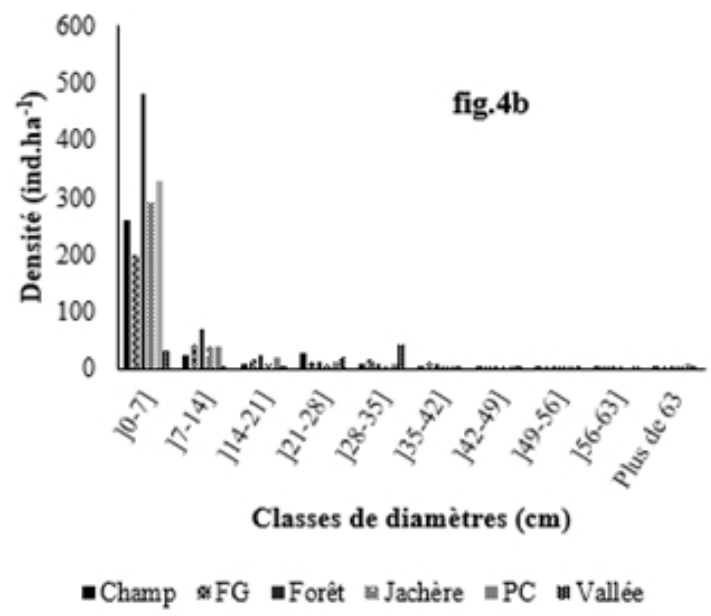

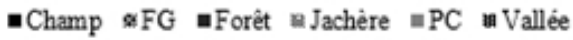

Figure 4. Structures de la végétation à Saré Yorobana selon le diamètre

\section{Structures de la végétation à Keur Birame}

La répartition des individus dans les classes de diamètres montre globalement sur le terroir villageois de Keur Birame une structure en L qui révèle un peuplement relativement jeune (fig.5a). La classe de diamètre ]0$7 \mathrm{~cm}$ ] est abondante mais la fréquence des individus diminue selon que le diamètre augmente. La répartition des classes de diamètres dans les faciès de végétation sur le terroir de Keur Birame (fig.5b) montre des structures plus irrégulières avec notamment l'absence d'individus des classes intermédiaires. Seules les diamètres $] 0-7 \mathrm{~cm}]$ et $] 7-14 \mathrm{~cm}$ ] sont représentées sur cette figure pour les arbres de petits diamètres contre les gros des classes $] 56-63 \mathrm{~cm}]$ et plus au niveau des champs. Dans les deux autres faciès, uniquement la classe de diamètre $] 0-7 \mathrm{~cm}]$ est visible.

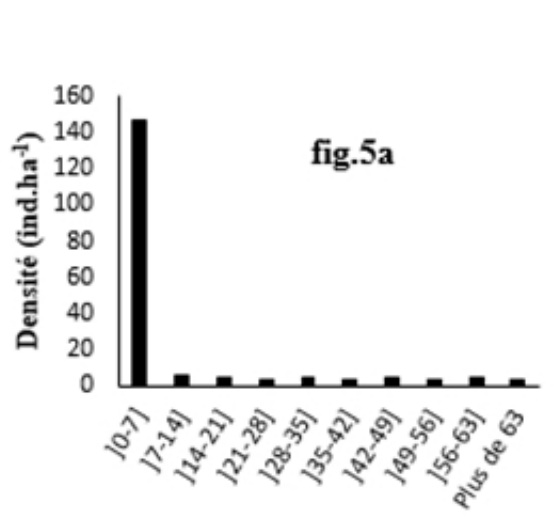

Classes de diamètres $(\mathrm{cm})$

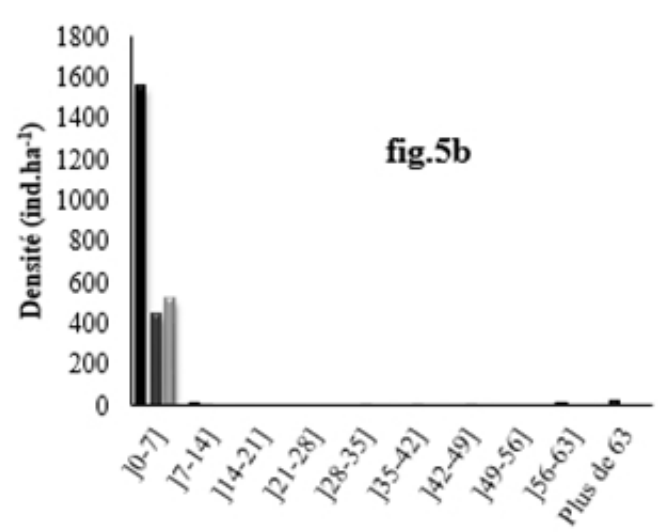

Classes de diamètres $(\mathrm{cm})$

Figure 5. Structures de la végétation à Keur Birame selon le diamètre 


\section{Discussion}

\section{Diversité floristique}

L'analyse globale de la flore des deux terroirs villageois révèle une richesse spécifique de 93 espèces appartenant à 80 genres et réparties en 33 familles botaniques. Toutefois, les deux sites appartiennent à des communautés floristiques différentes. Saré Yorobana renferme 78 espèces contre 45 espèces à Keur Birame. Les familles dominantes à Saré Yorobana sont celles des Caesalpiniaceae, des Anacardiaceae, des Fabaceae et des Combretaceae. Tandis qu'à Keur Birame, les familles prédominantes sont les Mimosaceae et les Caesalpiniaceae. Dans la zone du Bassin arachidier, Mbow (2008) a recensé 48 espèces de 41 genres répartis en 26 familles dont les plus représentées sont celles des Combretaceae, des Anacardiaceae et les Rubiaceae. Cependant, bien qu'ils soient soumis à des climats différents, les champs des deux localités renferment chacun 38 espèces avec une prédominance des Combretaceae. Faye et al. (2002) avaient observé 39 espèces dans les parcelles cultivées du village de Sobouldé en Haute Casamance au Sénégal. Par ailleurs, à Keur Birame, malgré l'impact des activités anthropiques sur le faciès champ, il demeure plus diversifié que le plateau cuirassé et la mise en défens. Mais il faut noter que dans ces milieux sous forte pressions foncière, l'activité anthropique est présente partout sous des formes différentes. La diversité plus élevée dans les champs s'explique par la présence en nombre important d'espèces se multipliant par rejets de souches en plus du fait que la mise en défens n'occupe qu'une bande large de moins de $200 \mathrm{~m}$ traversant le terroir mais également au milieu de vie hostile pour les plantes qu'est le plateau cuirassé. En effet Donfack (1998), montre que la rareté ou la disparition des espèces peut être due à la non-adaptation aux conditions du milieu, à l'inhibition de la germination des graines ou à l'absence de rejets.

\section{Paramètres structuraux}

\section{Surface terrière et taux de recouvrement}

La surface terrière $\left(2,70 \mathrm{~m}^{2} \cdot \mathrm{ha}^{-1}\right)$ et le taux de recouvrement $(7,32 \%)$ à Keur Birame sont moins importants qu'à Saré Yorobana $\left(4,39 \mathrm{~m}^{2} \cdot \mathrm{ha}^{-1}\right.$ et $20,90 \%$ respectivement). Ce qui fait état d'une part de la présence d'un peuplement épars et d'autre part d'une prédominance d'individus de petits diamètres. Ces deux paramètres sont encore plus faibles au niveau de la mise en défens $\left(0,37 \mathrm{~m}^{2} \cdot \mathrm{ha}^{-1}\right.$ et 2,68 \%) et du plateau cuirassé $\left(0,40 \mathrm{~m}^{2} \cdot\right.$ ha $^{-1}$ et 2,26 $\%)$ du terroir de Keur Birame. Ceci peut s'expliquer entre autres par la prédominance des Combretaceae et par l'hostilité du milieu (roche affleurante au niveau du plateau cuirassé) qui ne favorise pas l'installation des grands arbres. À Saré Yorobana, la situation est moins délicate du point de vue du recouvrement végétal surtout au niveau de la forêt galerie $(35,82 \%)$ mais en 
ce qui est de la surface terrière elle demeure faible atteignant sa plus forte valeur $\left(6,54 \mathrm{~m}^{2}\right.$. ha-1) dans la forêt galerie. La relative importance de la surface terrière au niveau des champs est due à la présence d'arbres de gros diamètre dans les parcs agroforestiers à Cordyla pinnata et Adansonia digitata.

\section{Taux de régénération}

Le taux de régénération très élevé à Keur Birame ainsi qu'au niveau des champs et des jachères de Saré Yorobana pourrait s'expliquer par le fait que les inventaires ont été réalisés après les récoltes au moment où les espèces qui avaient été coupées pour les besoins des cultures ont commencé à rejeter. Cependant, le faible taux de régénération $(1,41 \%)$ de la forêt à Saré Yorobana est dû au passage du feu de brousse avant la réalisation de l'inventaire, ce qui a décimé la régénération.

\section{Densité et structure}

À l'échelle des terroirs, la densité réelle est plus importante à Saré Yorobana (322 ind.ha ${ }^{-1}$ ) qu'à Keur Birame (139 ind.ha $\left.{ }^{-1}\right)$. En considérant les faciès de végétation des deux terroirs, seule la forêt (590 ind. ha ${ }^{-1}$ ) est plus dense que la mise en défens (450 ind. ha ${ }^{-1}$ ). Cette situation pourrait s'expliquer par l'abondance des rejets de Combretaceae au niveau de la mise en défens à Keur Birame. Les unités cultivées des deux terroirs ont les plus faibles densités réelles du fait des défrichements et des mauvaises pratiques agricoles.

La structure horizontale du peuplement présente une allure en « $\mathrm{L}$ » caractéristique d'un peuplement jeune avec un important taux de régénération aussi bien à Saré Yorobana qu'à Keur Birame. Toutefois, à l'échelle des faciès, des problèmes subsistent notamment dans les champs où le renouvellement végétal est compromis par les défrichements répétés. Ces résultats sont similaires à ceux de Diédhiou et $a l$. (2014) selon qui les problèmes de régénération dans le terroir insulaire de Mar Fafaco seraient dûs au manque de suivi et de protection des jeunes pousses contre les animaux et lors des défrichements. Dans le même sens, Yaméogo et al. (2013) ont montré que les arbres du parc étudié au Burkina Faso sont principalement utilisés pour satisfaire les besoins alimentaires et médicinaux (humains et animaux) tout en reconnaissant le faible taux de régénération des espèces qui composent les parcs agroforestiers. Cependant, les densités élevées au niveau des structures ne sont que des jeunes pousses qui sont systématiquement coupées à la prochaine saison culturale. Par ailleurs, à Saré Yorobana, la quête de nouvelles terres de cultures amène la population à empiéter sur la forêt mais également les coupes de bois de chauffe et/ou de services. 


\section{Conclusion}

La présente étude a permis de caractériser la diversité floristique des terroirs villageois de Saré Yorobana et Keur Birame à travers des relevés de végétation au niveau de leurs différents faciès. Cette dernière est de 93 espèces appartenant à 80 genres et réparties en 33 familles botaniques sur les deux terroirs villageois. Cependant, Saré Yorobana situé en zone soudanoguinéenne est floristiquement plus riche et compte 79 espèces contre 45 espèces pour Keur Birame en zone soudanienne. Les structures font état en apparence d'une végétation qui se régénère bien mais en réalité, considérant séparément les faciès, cela cache un réel problème de renouvellement des peuplements végétaux lié aux défrichements pour les besoins de l'agriculture (i), des coupes pour le bois de chauffe et/ou de service (ii) et des feux de brousse (iii) principalement à Saré Yorobana. Des efforts devraient être consentis pour le maintien des arbres surtout dans les unités cultivées pour une meilleure conservation de la biodiversité et l'amélioration du cadre de vie.

\section{References:}

1. Arbonnier, M. (2009). Arbres, arbustes et lianes des zones séches d'Afrique de l'Ouest. Edition Quae troisième édition. 576 p.

2. Baccini, A. (2010). Statistique Descriptive Multidimensionnelle. Publications de l'Institut de Mathématiques de Toulouse. UMR CNRS 5219 Université Paul Sabatier - 31062 - Toulouse cedex 9. 33p.

3. Berhaut, J. (1967). Flore du Sénégal. 2ème Ed. Clairafrique, Dakar, $485 \mathrm{p}$.

4. Berhaut, J. (1988). Flore illustrée du Sénégal. Clairafrique, Dakar, Thome I-IX.

5. Breman, H. \& Kessler, J.J. (1995). Woody Plants in Agro-Ecosystems ofSemi-Arid Regions, with an Emphasis on thelSahelian Countries, Berlin-Heidelberg, Springer-Verlag, 340 p.

6. CE-FAO (2000). Etude sur les ressources forestières et les plantations forestières du Sénégal. Période : 1992- 99. Rapport, juin. 61 p.de Wolf, J. (1998). Species composition and structure of the woody vegetation of the Middle Casamance region (Senegal). Forest Ecology and Management 111 (2-3), 249-264.

7. Diédhiou, M. A. A., Faye, E., Ngom, D. \& Touré, M. A. (2014). Identification et caractérisation floristiques des parcs agroforestiers du terroir insulaire de Mar Fafaco (Fatick, Sénégal) J. Appl. Biosci, 79:6855 - 6866 .

8. Donfack, P. (1998). Végétation des jachères du Nord-Cameroun : typologie, diversité, dynamique, production. Thèse de doctorat d'Etat, Université de Yaoundé 1, Faculté des sciences, Mention Biologie et Physiologie végétale, Yaoundé, 225 p. 
9. FAO (2010). Evaluation des ressources forestières mondiales. Rapport national, Sénégal.

10. Faye, E. (2005). Étude floristique, phytosociologique, phytogéographique, ethnobotanique, et de la régénération après coupe des ressources ligneuses dans la région de Kaolack (Sénégal). DEA Université Libre de Bruxelles, 83 p.

11. Faye, E., Diallo, H., Samba, S.A.N., Touré, M.A., Dramé, A., Fall, B., Lejoly, J., Diatta, M., Kaïré, M., De Canniere, C., Mahy, G. \& Bogaert, J. (2013). Importance de la méthode de coupe sur la régénération de Combretaceae du Bassin arachidier sénégalais. TROPICULTURA, 31(1) 44-52.

12. Faye, E., Masse, D. \& Diatta, M. (2002). Dynamique de la régénération ligneuse durant la phase de culture dans un système de culture semi-permanente du sud du Sénégal. In Actes du colloque, mai 2002, Garoua, Cameroun. Prasac, N’Djamena, Tchad - Cirad, Montpellier, France.

13. Frontier, S. \& Pichod-Viale, D. (1995). Écosystèmes : structure, fonctionnement, évolution. Masson, $447 \mathrm{p}$.

14. Lebrun, J. P. \& Stork, A. L. (1991). Énumération des plantes à fleurs d'Afrique tropicale. Conservation du jardin botanique de Genève, Vol I : $249 \mathrm{p}$.

15. Lebrun, J. P. \& Stork, A. L. (1992). Énumération des plantes à fleurs d'Afrique tropicale. Conservation du jardin botanique de Genève, Vol II : $257 \mathrm{p}$.

16. Lebrun, J. P. \& Stork, A. L. (1995). Énumération des plantes à fleurs d'Afrique tropicale. Conservation du jardin botanique de Genève, Vol III : $341 \mathrm{p}$.

17. Lebrun, J. P. \& Stork, A. L. (1997). Énumération des plantes à fleurs d'Afrique tropicale. Conservation du jardin botanique de Genève, Vol IV : $712 \mathrm{p}$.

18. Manley, R. (2000). Dynamique de la matière organique à l'échelle d'un terroir agro-pastoral de savane ouest-africaine (Sud-Sénégal). Thèse, ENGREF. Montpellier, 326p.

19. May, R. M. (1990). Living in Patchy Environment. Oxford University Press; 1-22.

20. Mbow, M. A. (2008). Importance de l'arbre dans les systèmes d'utilisation des terres dans le sud-ouest du bassin arachidier au Sénégal. Mémoire de DEA, BV-UCAD, 44 p.

21. Ndiaye, ND. (1999). Les nématodes du sol dans les systèmes de cultures à jachères du sénégal: dynamique des peuplements et relations avec les facteurs abiotiques et biotiques telluriques. Thèse, FST/UCAD. Dakar, 170 p. 
22. Ndiaye, I., Camara, B., Ngom, D. \& Sarr, O. (2017). Diversité spécifique et usages ethnobotaniques des ligneux suivant sun gradient pluviométrique Nord-Sud dans le bassin arachidier sénégalais J. Appl. Biosci, 113: 11123-11137.

23. Ngom, D. (2013). Diversité végétale et quantification des services écosystèmiques de la réserve de biosphère du Ferlo (Nord-Sénégal). Thèse, ED-SEV/UCAD. Dakar, 167p.

24. Noss, R. F. (1990). Can we maintain biological and ecologicalintegrity? Conservation Biology, 4: 241-243.

25. Poupon, H. (1980). Structure et dynamique de la strate ligneuse d'une steppe sahélienne au nord du Sénégal. ORSTOM éd. (Etudes \& Thèses), Paris : $307 \mathrm{p}$.

26. Ramade, F. (2003). Eléments d'Ecologie: Ecologie fondamentale. $3^{\text {ème }}$ édition, Dunod, Paris; 690 p.

27. Roberts-Pichette, P. \& Gillespie, L. (2002). Protocole de suivi de la biodiversité végétale terrestre. Le réseau d'évaluation et de surveillance écologiques Canada. Sur http://www.eman.ese.ca/rese/ecotools/terrestrial/vegetation/glossary. $\mathrm{html}$

28. Sarr, O., Diatta, A.S., Gueye, M., Ndiaye, P.M., Guissé, A. \& Akpo, L.E. (2013). Importance des ligneux fourragers dans un système agropastoral au Sénégal (Afrique de l'ouest). Revue Méd. Vét. Toulouse, 164 (1) : 2-8.

29. Sarr, O., Ngom, D., Ngaryo, F.T., Diatta, S. \& Akpo, L. E. (2014). Etat de la végétation ligneuse dans trois unités d'utilisation des terres d'une zone agropastorale au Sénégal (région de Kaffrine). Int. J. Biol. Chem. Sci. 8(6): 2669-2684.

30. Yaméogo, J.T., Some, A.N., Mette Lykke, A., Hien, M. \& Nacro, H.B. (2013). Restauration des potentialités de sols dégradés à l'aide du zaï et des cordons pierreux à l'Ouest du Burkina Faso. TROPICULTURA, 31, 4, 224-230. 